\title{
Ingeniería de Servidores desde la perspectiva de un estudiante
}

\author{
Jorge Chamorro Padial \\ Grado en Ingeniería Informática. E.T.S. Ing. Informática y de \\ Telecomunicación, Universidad de Granada \\ Granada, España \\ jorgechp@correo.ugr.es
}

\begin{abstract}
Resumen. Ingeniería de Servidores es una asignatura que se imparte en el tercer año del Grado en Ingeniería Informática. En este artículo se analiza, desde el punto de vista de un estudiante, la evolución de la asignatura. La metodología y el sistema de evaluación son descritos y examinados con el fin de aportar una valoración de debilidades y fortalezas observadas tanto por el autor como por estudiantes de otros grupos cuya opinión queda reflejada por medio de una pequeña encuesta.
\end{abstract}

Palabras Clave: Ingeniería de Servidores, Docencia, Motivación del estudiante

\begin{abstract}
Ingeniería de Servidores (English: Server Engineering) is a Computer Engineering subject taught on the third year of Computer Engineering degree. In this paper, the progress of the subject is analyzed from a student's point of view. Methodology and the evaluation system are described and examined in order to provide a feedback of weakness and strengths observed so much for the author as another students of different groups whose opinion is reflected through a survey.
\end{abstract}

Keywords: Server Engineering, Teaching, Student motivation

\section{Introdución}

Ingeniería de Servidores (en adelante, ISE) es una asignatura obligatoria de formación específica de rama que se imparte en el quinto semestre (tercer curso) del Grado en Ingeniería Informática.

El temario teórico impartido en la asignatura se muestra a continuación:

1. Introducción a la Ingeniería de Servidores

2. Componentes hardware de un servidor

3. Monitorización de servicios y programas 
4. Análisis comparativo de rendimiento (benchmarking)

5. Análisis operacional en servidores

6. Exposición de casos de estudio ${ }^{1}$

En cuanto al temario práctico, se distribuye de la siguiente manera:

1. Instalación virtualizada de $\mathrm{SO}$ en servidores

2. Instalación y configuración básica de servicios

3. Monitorización de la actividad de un servidor

4. Benchmarking de servidores

5. Optimización del rendimiento de servidores

Además, se imparten dos seminarios ("Administración y mantenimiento de Servidores" y "Elaboración de un pliego de condiciones técnicas"). Todo esto, junto a un Trabajo autónomo que se elabora durante el transcurso del semestre, configuran la estructura de la asignatura y permiten alcanzar los objetivos recogidos en la Guía Docente de la misma. (Disponible en la web de Grados de la UGR: http://grados.ugr.es/informatica/pages/infoacademica/guias-docentes )

La evaluación de las diferentes partes de la asignatura se realiza de la siguiente manera:

- En convocatoria ordinaria:

- Teoría: ponderada al $40 \%$ de la nota final. La valoración de esta parte se determina por medio de un exámen teórico.

- Práctica: ponderada al 30\% de la nota final. Incluye la evaluación de las memorias de cada tema, los dos seminarios y un examen de prácticas.

- Trabajo: ponderado al $30 \%$ de la nota final.

- En la convocatoria extraordinaria:

- Teoría: pondera al $40 \%$ de la nota. Se valora por medio de un examen teoórico.

- Trabajos a entregar: ponderan el 30\% de la nota.

- Examen de prácticas y seminarios: Ponderan al 30\% de la nota.

En todas las partes es necesario obtener, al menos, un 4 sobre 10 para poder obtener una calificación final positiva.

A continuación, se analizará y valorará, en sus diferentes partes, el desarrollo de la asignatura narrando la experiencia del autor como estudiante así como la de otros compañeros durante el curso 2013/2014.

1 El Tema 6, por motivos de falta de tiempo no se ha podido impartir en todos los grupos. 


\section{Desarrollo de la asignatura}

\subsection{Teoría}

¿Cómo obtener el rendimiento más alto con el coste más pequeño?. Esta es una de las cuestiones principales que desde ISE se trata de responder. Para ello, el primer tema de la asignatura trata de introducir y aclarar conceptos básicos sobre Sistemas Informáticos y sus diferentes clasificaciones, paralelismo, fundamentos de la Ingeniería de Servidores, medidas de rendimiento y comparación de prestaciones y la Ley de Amdahl. En realidad, estos conceptos no se introducen por primera vez en esta asignatura sino que se realiza un breve repaso y se toma como punto de partida el conocimiendo adquirido en asignaturas como Estructura de Computadores, Sistemas Concurrentes y Distribuídos y, especialemente, de Arquitectura de Computadores. Esta última asignatura se podría considerar como el punto de partida de ISE.

El tema de introducción resulta importante de cara al resto de la asignatura ya que permite asentar conocimientos que, si bien ya se suponen adquiridos en otras asignaturas, no se han estudiado con el enfoque o la profundidad necesaria para ISE.

A pesar de que existen conceptos como el de rendimiento o como la Ley de Amdahl, que se estudian en Arquitectura de Computadores, no es hasta ISE cuando esos conocimientos se asimilan definitivamente y el estudiante logra ver la utilidad de los mismos.

Una vez se han establecido los cimientos de la asignatura, se da el salto a la Monitorización. ¿Cómo medir el rendimiento de mi servidor?, es la pregunta a la que da respuesta este tema que comienza hablando de monitores, tipos de monitores, funcionamiento de los mismos y sus atributos (exactitud, precisión, frecuencia de muestreo, sobrecarga que generan...) para saltar al uso de las herramientas que nos proporciona Unix y alternativas a estas como SarCheck (http://www.sarcheck.com/) .

Aunque no es condición indispensable para asimilar positivamente este tema, es importante que el alumnado haya superado las asignaturas de Fundamentos del Software y la de Sistemas Operativos, donde ya en su parte práctica se ha trabajado con la mayor parte de las herramientas de monitorización del Sistema Operativo además de hablar a más bajo nivel de los procesos del Sistema y su funcionamiento.

Realmente, la segunda parte del tema, que trata de la Monitorización a nivel de aplicaciones (profilers) es la que aporta nuevos conocimientos al estudiante que no se han impartido en asignaturas anteriores. Puede resultar llamativo que hayan tenido que transcurrir tres cursos en una carrera donde, en prácticamente en cada asignatura impartida hasta entonces, ha sido necesario programar, para que el estudiante tenga su primer contacto con los profilers. Si bien es cierto que en primero se utilizó Valgrind en una asignatura, no se explotó el verdadero potencial que tiene este programa y cabe preguntarse si sería necesario que los estudiantes del Grado se familiarizaran con este tipo de herramientas cuanto antes mejor, del mismo modo que se hace con los depuradores. 
Una vez que sabemos qué es el rendimiento y cómo medirlo, llega el momento de preguntarnos: ¿Qué servidor tiene mejor rendimiento?. La comparación de diferentes sistemas por medio de índices, benchmarks y un análisis de sus resultados suponen los conocimientos a adquirir en este tema en el que, además, se plantea acabar con los mitos de la existencia de un "índice perfecto" que permita compararlo todo. El tercer tema culmina con una pequeña introducción al diseño de experimentos para la comparación de servidores. Quizás, la mayor dificultad de este tema radica en el uso de la Estadística, necesaria para comprobar si las diferencias entre las mediciones realizadas a varios sistemas son o no significativas. Si bien el nivel requerido al estudiante se adquiere en la asignatura de Estadística que se imparte en el segundo semestre, suele ser común que los estudiantes en tercero sigan arrastrando asignaturas de primero o bien dejen estas para el final de la carrera.

Terminado el tercer tema, entramos en el que tema que, probablemente, da mayor sentido a la asignatura: El análisis operacional de servidores. Los conceptos que aquí se estudian son nuevos y no se han visto en ninguna otra asignatura: La abstracción respecto a los servidores, que son representados con diferentes modelos de Sistemas Informáticos según su comportamiento con un conjunto de variables que les afectan y permiten sus análisis gracias a las Leyes Operacionales que rigen a dichas variables. Es en este tema donde se estudia qué es la hipótesis de equilibro de flujo, la Ley de Little, Ley de la Utilización, Ley del flujo forzado y la Ley general del tiempo de respuesta. A continuación, hacen análisis asintóticos para conseguir descubrir los cuellos de botella y las limitaciones en el rendimiento. En definitiva, la pregunta que nos hacemos esta vez es ¿Cómo modelar el rendimiento y predecir la capacidad de mi servidor?

Tras un tema que quizás termina siendo algo denso, el siguiente de la asignatura podría catalogarse de "cultura general" que todo Ingeniero Informático debería conocer: Componentes hardware de un servidor. En este tema, se estudia qué es una Placa Base y sus componentes. Es interesante señalar que el enfoque que se le dio en clase de Teoría no fue meramente una lección teórica formal sino que fue acompañado con ejemplos de modelos de componentes obtenidos directamente desde la web del fabricante. Dando al estudiante una perspectiva más realista sobre las prestaciones y el precio actual en el cual oscilan esos componentes, así como cuáles son los principales fabricantes de estos componentes. En definitiva, responder a la pregunta ¿Qué hardware es el más adecuado para mi servidor?.

Finalmente, el último tema enlaza con el Trabajo de la asignatura. Algunos estudiantes han expuesto sus temas a compañeros de grupo con el fin de que estos puedan aprender sobre otras áreas que ellos no han trabajado. Por problemas de tiempo, en el grupo del autor no se pudo llegar a realizar estas exposiciones. Por lo que se ha realizado una pequeña encuesta en la que han participado cuatro estudiantes de este curso y del curso anterior:

- $\quad$ Pregunta 1: ¿Sobre qué hiciste tu trabajo de ISE?

Estudiante 1: Balanceo de carga y planificación de servidores.

Estudiante 2: Hice el trabajo sobre servidores de alojamiento de archivos.

Estudiante 3: Sobre refrigeración de centros de datos. Donde se explicaban las diferentes formas de refrigeración actuales con sus ventajas e inconvenientes. 
- Estudiante 4: Clustering, aunque lo quería hacer sobre free cooling.

- $\quad$ Pregunta 2: ¿Crees que el trabajo te motivó para investigar y aprender sobre este tema?

- Estudiante 1: Si que tuvimos que investigar ya que no sabíamos apenas del tema (algo de planificación si, por Sistemas Operativos, pero muy básico). La motivación fue, sobretodo, hacer un buen trabajo y hacerlo bien.

- Estudiante 2: Sí, ya que los servidores de alojamiento de archivos representan un papel fundamental para compartir información de manera cómoda. Creo que conocer los avances tecnológicos en este campo y los sistemas que se desarrollan es importante. Como por ejemplo el caso de Dropbox, que permite alojar un gran número de ficheros de forma rápida siguiendo el modelo de almacenamiento en la nube. A su vez también es importante conocer como afecta el marco legal a estos sistemas, ya que los desarrolladores de algunos de estos sistemas han llegado a recibir acusaciones por infracción de derechos de autor. Siendo uno de los casos más conocidos el de Megaupload.

- Estudiante 3: El trabajo me resultó muy interesante y me motivo para investigar más sobre nuevas tecnologias para la refrigeracion.

- Estudiante 4: No, para nada.

- Pregunta 3: (solo si la tercera pregunta tiene respuesta afirmativa) ¿En tu grupo se hicieron exposiciones?

- Estudiante 1: Sí, se hicieron.

- Estudiante 2: Sí, se hicieron exposiciones.

- Estudiante 3: Sí.

- Estudiante 4: Sí

- Pregunta 4: ¿Expusiste tu trabajo en clase?

- Estudiante 1: No, no dio tiempo.

- Estudiante 2: No, ya que mi clase estaba formada por un gran número de alumnos y no pudimos exponer todos.

- Estudiante 3: Sí.

- Estudiante 4: No.

- $\quad$ Pregunta 5: ¿Crees que las exposiciones de otros compañeros te ayudaron a aprender e interesarte sobre otros temas relacionados con la Ingeniería de Servidores?

- Estudiante 1: Siempre que expone un alumno hay riesgo de que la exposición no sea muy buena y el tema se haga pesado, pero si que aprendimos.

- Estudiante 2: Sí, ya que hubo una variedad de tecnologías importante. Mediante los trabajos de exposición de los compañeros pude conocer aspectos interesantes de estas tecnologías relacionadas con la Ingeniería de Servidores que desconocía hasta el momento.

- Estudiante 3: Había exposiciones de temas muy interesantes de los cuales no conocía nada sobre ellos, y me instaron a buscar mas información.

- Estudiante 4: No fuí a la de otros compañeros. Todo lo relacionado con servidores me parece aburrido.

\subsection{Práctica}

En la parte práctica de la asignatura, al estudiante se le sitúa en el rol de un administrador de sistemas que debe configurar y mantener un servidor. Se trabaja tanto en Windows Server como en GNU/Linux (Ubuntu Server y CentOS 
principalmente, aunque también se pueden utilizar otras distribuciones como openSUSE).

Como instalar tantos Sistemas Operativos con el único fin de probarlos no es una situación ideal, las prácticas se realizan virtualizando estos Sistemas Operativos mediante VMware o VirtualBox. De esto trata el primer tema, donde se introducen conceptos como Máquina Virtual o virtualización. Tras esto, se comienza la instalación de los diferentes Sistemas Operativos creando RAIDs y usando volúmenes lógicos cifrados. Finalmente, se prueba la disponibilidad del servidor frente a fallos del RAID. Es decir, se realiza una instalación y configuración habitual en un entorno real de trabajo.

Una vez tenemos instalados nuestros distintos servidores, toca instalar diferentes servidores, tales como ssh, ftp, apache... Tareas que se realizan en la segunda práctica.

La tercera práctica permite al estudiante probar diversas herramientas para monitorización como Ganglia, Nagios, Munin, Perfmon, Awstats, Cactis así como profilers, concretamente gprof y Valgrind. Esta práctica, si bien es útil de cara al futuro, termina resultando bastante pesada y larga por la gran cantidad de programas que hay que instalar y probar. Quizás sería recomendable centrar la atención en una cantidad menor de programas y usarlos más a fondo en vez de recorrer un amplio espectro de alternativas de forma superficial. De hecho, varios compañeros tuvieron problemas para entregar esta práctica y la siguiente debido a la carga de trabajo que requieren y a que su periodo de realización coincidía con el de prácticas de otras asignaturas de tercero que también requerían bastante tiempo. Finalmente, se instalan $\mathrm{y}$ prueban diferentes profilers.

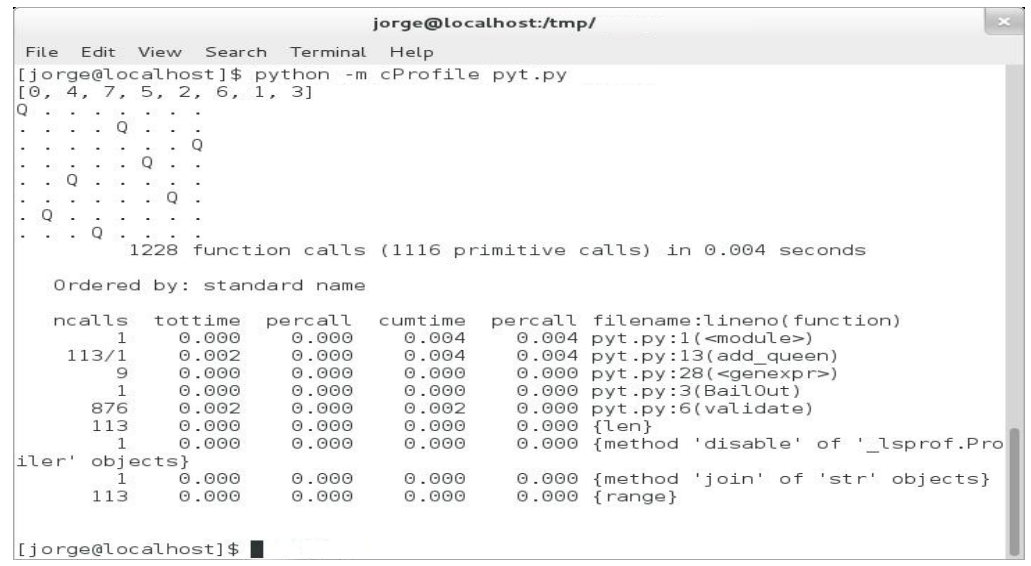

Figura1. Ejecución de la librería Cprofile (http://docs.python.org/2/library/profile.html) sobre una implementación del problema de las n-reinas como parte de un ejercicio de prácticas.

La cuarta práctica trata sobre benchmarks y test de estrés. Sigue una estructura similar a la anterior práctica: se prueban diferentes benchmarks tales como el Phoronix Suite, Jmeter, Siftsoftware Sandra y Aida64. Al final de la practica, el estudiante puede programar su propio benchmark.

Tras dos prácticas que, como se ha mencionado anteriormente, resultan algo densas, la última práctica de la asignatura trata la optimización de los servidores para 
mejorar sus prestaciones. Básicamente, consiste en mejorar el Servidor Web IIS, Apache, modificar parámetros del kernel de GNU/Linux y del registro de Windows y optimizar un servicio del sistema a elección del Estudiante.

\subsection{Seminarios}

Por último, durante las horas de prácticas se han impartido dos seminarios. El primero de ellos trata sobre Administración de Servidores y recorre diversos temas, empezando una introducción a las diferentes certificaciones profesionales que existen para continuar con una visión a las ofertas de empleo para administradores existentes hoy en día y terminando con buenas prácticas en la administración de servidores, ética, seguridad y una breve introducción a Windows PowerShell.

El segundo seminario introduce al estudiante en la legislación actual sobre pliegos. Ambos seminarios aportan conocimientos que todo estudiante del Grado debería conocer. Una vez más, ISE actúa como "cajón de sastre" donde se imparten contenidos que no necesariamente están totalmente relacionados con la asignatura (en alusión al segundo seminario).

\subsection{Trabajo Grupal/Autónomo}

El trabajo que el estudiante debe ir realizando a lo largo de la asignatura versa sobre la mejora de uno de los factores (disponibilidad, eficiencia energética, fiabilidad, mantenimiento, coste, escalabilidad, seguridad y extensibilidad), que contribuyen en la mejora del diseño o configuración de los servidores. El trabajo se divide en dos fases:

- Primera fase: Por equipos de hasta nueve personas. Se buscan y discuten sobre tecnologías y sistemas que permitan mejorar alguno de los factores anteriormente mencionados. Se fomenta en todo momento que se formen grupos grandes, ya que el número de miembros no influye en la calificación de esta fase. Pero los grupos con un gran número de integrantes tendrán ventaja a la hora de encontrar más soluciones y, por lo tanto, más posibilidades de obtener una mayor calificación. Desde mi punto de vista, esta política es acertada ya que así se consigue que cada estudiante, a nivel individual, obtenga información sobre un mayor número de soluciones que las que podría buscar de forma individual o en un grupo reducido. En concreto, en nuestro grupo de trabajo encontramos las siguientes soluciones:

Tabla 1. Soluciones aportadas por un grupo de trabajo para trabajar sobre los diferentes factores implicados en el diseño y/o configuración de servidores

\begin{tabular}{|c|cl|}
\hline Solución & Factores implicados \\
\hline & $\bullet$ & Rendimiento \\
RAID & $\bullet$ & Disponibilidad \\
\hline
\end{tabular}




\begin{tabular}{|c|c|}
\hline & - $\quad$ Coste \\
\hline SAI & $\begin{array}{ll}\text { - } & \text { Disponibilidad } \\
\text { - } & \text { Fiabilidad } \\
\text { - } & \text { Seguridad } \\
\text { - } & \text { Coste } \\
\end{array}$ \\
\hline Arquitectura ARM & - $\quad$ Eficiencia energética \\
\hline Bioclimatización & $\begin{array}{ll}\text { - } & \text { Eficiencia energética } \\
\text { - } & \text { Coste }\end{array}$ \\
\hline $\begin{array}{l}\text { Virtualización por } \\
\text { hardware }\end{array}$ & $\begin{array}{ll}- & \text { Coste } \\
\text { - } & \text { Escalabilidad } \\
\text { - } & \text { Disponibilidad }\end{array}$ \\
\hline Proxy inverso & - $\quad$ Seguridad \\
\hline Iptables & - $\quad$ Seguridad \\
\hline VPN & - $\quad$ Seguridad \\
\hline Rack TS IT & $\begin{array}{ll}- & \text { Eficiencia } \\
\text { - } & \text { Matenimiento }\end{array}$ \\
\hline Balanceo de carga & - $\quad$ Rendimiento \\
\hline Cifrado de datos & $\begin{array}{ll}- & \text { Seguridad } \\
- & \text { Disponibilidad }\end{array}$ \\
\hline Cloud Hosting & $\begin{array}{ll}\text { - } & \text { Rendimiento } \\
\text { - } & \text { Disponibilidad } \\
\text { - } & \text { Mantenimiento }\end{array}$ \\
\hline $\begin{array}{l}\text { Herramientas de } \\
\text { administración y } \\
\text { monitorización }\end{array}$ & $\begin{array}{ll}\text { - } & \text { Rendimiento } \\
\text { - } & \text { Disponibilidad } \\
\text { - } & \text { Mantenimiento }\end{array}$ \\
\hline Copias de seguridad & $\begin{array}{ll}- & \text { Seguridad } \\
\text { - } & \text { Disponbilidad }\end{array}$ \\
\hline Granjas de servidores & $\begin{array}{ll}- & \text { Rendimiento } \\
\text { - } & \text { Disponibilidad }\end{array}$ \\
\hline Blade (Servidor) & - $\quad$ Eficiencia energética \\
\hline $\begin{array}{l}\text { Algoritmos genéticos } \\
\text { aplicados a servidores }\end{array}$ & $\begin{array}{ll}- & \text { Rendimiento } \\
\text { - } & \text { Eficacia } \\
\text { - } & \text { Coste }\end{array}$ \\
\hline $\begin{array}{l}\text { Refrigeración líquida } \\
\text { de servidores }\end{array}$ & $\begin{array}{ll}\text { - } & \text { Coste } \\
\text { - } & \text { Disponibilidad }\end{array}$ \\
\hline
\end{tabular}

Todos estas soluciones fueron sometidas a una puesta en común entre los miembros del grupo. Por lo que, de una forma simple, cada estudiante ha podido adquirir unas nociones sobre la existencia de estas soluciones y en qué consiste cada una de ellas. De una de estas soluciones, cada miembro el grupo desarrollará la parte autónoma del trabajo. No existen dos trabajos sobre una misma solución. De manera que gran parte de estas soluciones al final serán investigadas y desarrolladas por los estudiantes a lo largo de la asignatura.

- Segunda fase: Como se ha mencionado anteriormente, cada miembro del grupo realiza un trabajo sobre una de las soluciones aportadas por el grupo. El objetivo es presentar tres documentos:

- Memoria: De hasta doce páginas, donde se plasma todo lo que se ha investigado acerca de la solución.

- Presentación: De cara a una posible exposición en clase.

- Test: Preguntas tipo test sobre la presentación realizada. 
En el caso del autor, el trabajo desarrollado fue sobre Arquitecturas de microprocesadores con el objetivo de conseguir eficiencia energética. Durante el mismo, se investigó sobre la demanda actual del mercado de procesadores de este tipo para pasar a continuación a un análisis de la arquitectura RISC y sus ventajas frente CISC en cuanto a consumo energético se refiere. Comparando ARM y su evolución histórica con la alternativa de Intel y sus procesadores Atom [1] [2]. Posteriormente, se realizó una comparativa con diferentes benchmarks y tests de estrés sobre ARM, Atom y modelos de otros fabricantes [3] y, finalmente, se analizaron las perspectivas de futuro de los procesadores de bajo consumo. Una vez terminado el trabajo, fue enviado a cuatro estudiantes al azar para que lo revisaran y evaluaran en base a una plantilla establecida por los profesores. La identidad del autor del trabajo nunca era conocida por la de los revisores, para asegurar la mayor imparcialidad posible. Del mismo modo, el autor puede ver las correcciones realizadas en su trabajo pero no puede ver quién se las hizo. Un estudiante corrige un total de tres trabajos. Con este Sistema, los trabajos se cruzan y cada estudiante, además de su propio trabajo, aprende sobre lo que sus compañeros de grupo han hecho y se toma en serio la lectura y la asimilación de contenidos de sus compañeros, puesto que luego deberá evaluarlos.

Finalmente, y en los grupos donde ha sido posible (porque no haya faltado tiempo de clase en dichos grupos), algunos trabajos han sido expuestos. Si analizamos con detalle este sistema. Podemos ver cómo cada estudiante, a lo largo de la asignatura:

- Ha buscado e investigado, al menos de forma superficial, sobre un gran número de diferentes soluciones para el diseño, configuración y mantenimiento de servidores.

- Ha aprendido de una manera completa acerca de algunas soluciones en concreto, expuestas por sus compañeros en clase.

- Conoce en profundidad tres soluciones, ya que ha tenido acceso y ha podido evaluar el trabajo de tres compañeros.

- Además, puede ponerse tanto en la piel de un profesor que debe evaluar trabajos y, a su vez, obtener retroalimentación con las opiniones de sus compañeros sobre los puntos fuertes y los puntos débiles de su trabajo. Propiciando la adquisición de competencias tranversales establecidas en el Grado. [5]

\section{Conclusiones}

ISE es una asignatura donde se combinan, de forma bastante acertada, conocimientos teóricos sobre la Ingeniería de Servidores y conocimientos prácticos sobre configuración y administración de los mismos con un enfoque práctico simulando la vida real de muchos Ingenieros que tienen que lidiar con la administración de sistemas. Como parte negativa, la práctica tercera y cuarta han 
requerido demasiado tiempo. El hecho de que, con un solo ejercicio sin realizar la memoria pueda calificarse de forma negativa, puede dar lugar a situaciones injustas, por lo que habría que plantearse una relajación de ese criterio de evaluación.

La parte teórica de la asignatura quizás podría reconfigurarse con el fin de menguar la curva de aprendizaje, ya que el penúltimo y especialmente el último tema contienen una gran cantidad de conceptos nuevos que deben ser asimilados en poco tiempo.

El trabajo incentiva a investigar sobre temas que resultan novedosos, y la posibilidad de acceder y aprovechar los trabajos de los compañeros es una forma de conseguir que el estudiante aprenda más y empleando menos tiempo del que necesitaría, por sí mismo, para investigar sobre cada una de las diferentes soluciones.

Por último, tanto los seminarios como el tema sobre componentes del hardware pueden servir como forma de motivación, ya que "rompen" la rutina a la vez que se imparten conocimientos necesarios para el estudiante. Este sistema podría ser exportado a otras asignaturas, consiguiendo así enriquecer la formación del alumnado.

Finalmente, cuando ha llegado el momento de preparar el examen de teoría, el estudiante tenía a su disposición, como material de apoyo, problemas cuya solución venía dada en la relación de ejercicios. Hay que tener en cuenta que, en época de exámenes, el estudiante se encuentra "solo" ante los apuntes de clase. Introducir soluciones en las relaciones de problemas permiten al estudiante medir su progreso y no navegar en un mar de dudas ("¿estoy aprendiendo correctamente o me estoy equivocando por completo?") y sirve como motivación ("lo que estoy aprendiendo lo aplico de forma correcta, merece la pena que siga preparándome la asignatura", "me he equivocado, pero ahora sé cómo tengo que hacerlo para que esté bien").

\section{Agradecimientos}

A Héctor Emilio Pomares Cintas y a Eloi Montilla i Busquets por la orientación y ayuda prestada a la hora de redactar este artículo.

A Isaac Morely Rodríguez, Natalia Romero Parra, Emilio Nieto Morilla, Germán Iglesias Padial y Fernando Palacios Lopez por su participación en este artículo aportando su visión y opiniones sobre ISE.

\section{Referencias}

1. Stokes, J.: RISC vs. CISC in the mobile era. arstechnica. http://arstechnica.com/gadgets/2008/05/risc-vs-cisc-mobile-era/ (2008)

2. Bichenn, S.: ARM to Intel: it's not just about the transistor. hexus.net. http://hexus.net/business/features/components/30305-arm-intel-its-just-transistor/ (2011)

3. Chak, J.C.: Benchmarks i mètriques d'avaluació de plataformes encastades multimèdia. Diposit de la Rercerca de Catalunya. Escola Tècnica Superior d'Enginyeria Secció d'Enginyeria Informàtica. Universitat Autònoma de Barcelona, http://www.recercat.net/bitstream/handle/2072/14087/PFC+Joan+Carles+Chak+Ma.pdf?sequence=1 (2008)

4. Schönborn, T.: SoC Shootout: x86 vs. ARM. NotebookCheck.net http://www.notebookcheck.net/SoC-Shootout-x86-vs-ARM.99496.0.html (2013)

5. Ministerio de Educación, Cultura y Deporte. Memoria del Grado en Ingeniería Informática. P6. http://grados.ugr.es/informatica/pages/infoacademica/archivos/verificaingenieriainformatica/\%21 